\title{
Ueber die quantitative Bestimmung der Spaltungsprodukte von Eiweisskörpern.
}

\author{
Von \\ Edwin Hart (Geneva, N.-Y.). \\ (Aus dem physiologischen Institut in Heidelberg.) \\ (Der Redaction zugegangen am 15. Juli 1901.)
}

Die früheren Untersuchungen über die Eiweisskörper und ihre Spaltungsprodukte haben sich wesentlich auf ihre elementare Zusammensetzung und auf qualitative Arbeiten über ihre Spaltungsprodukte beschränkt. Erst in der letzten Zeit ist den quantitativen Ergebnissen und Methoden auf diesem Gebiete ein grösseres Interesse zugewendet worden, und besonders versprechen die von A. Kossel und F. Kutscher ${ }^{1}$ ) bei ihren letzten Arbeiten angewandten Methoden zur Bestimmung der Hexonbasen einen Aufschluss über das Verhältniss der verschiedenen Eiweisskörper zueinander. 'Von besonderem Werthe werden diese Methoden für die Erforschung der ersten Spaltungsprodukte der Eiweisskörper sein, denn die Frage nach der Natur der als Propeptone, Albumosen, Peptone bezeichneten Körper kann nur durch die quantitative Bestimmung ihrer Spaltungsprodukte in entscheidender Weise gefördert werden. Die Elementaranalysen von Möhlenfeld, Kistiakowsky, A. Kossel, Kühne und Chittenden, sowie von den Schülern des letztgenannten Forschers lehren, dass diese Produkte im Allgemeinen ärmer an Kohlenstoff und Stickstoff und reicher an Saverstoff sind als die ursprünglichen Eiweisskörper, und dass diese Veränderungen bedingt sind durch den Eintritt der Elemente des Wassers in das Eiweissmolekül. Durch die Ergebnisse der Elementaranalyse ist aber nicht entschieden, ob

1) Diese Zeitschrift, Bd. XXXI, S. 165. 
bei dieser hydrolytischen Umwandlung der Eiweisskörper auch zugleich ein Zerfall in mehrere verschiedenartige Antheile des Moleküls stattfindet. Diese Frage ist erst in neuerer Zeit von Pick, 1) $\mathrm{Haslam}^{2}$ ) und Andern in Angriff genommen worden. Pick zog aus seinen Untersuchungen den Schluss, dass die Heteroalbumose, die er nach seinem eigenen Verfahren darstellte, eine andere Constitution besitzen müsse, als die übrigen gleichzeitig in dem Verdauungsgemisch des Eiweisses vorgefundenen Albumosen. Haslam untersuchte ein Produkt, welches zwar denselben Namen führt, aber offenbar von Pick's Heteroalbumose völlig verschieden ist - nämlich die nach Kühne's Verfahren dargestellte «Heteroalbumose». Pick's Resultate lassen sich dahin zusammenfassen, dass die Heteroalbumose und die Protalbumose sich durch das Fehlen der durch $\alpha$-Naphthol und Schwefelsäure nachweisbaren furfurolbildenden Gruppe auszeichnen, dass die Heteroalbumose Glycocoll enthält, hingegen die Protalbumose nicht, dass fernerhin die Heteroalbumose wenig Tyrosin und Indol liefert, hingegen die Protalbumose viel von diesen Atomgruppen enthält. Ausserdem ist nach Pick die Menge des Stickstoffs der durch Phosphorwolframsäure fällbaren Substanzen bei der Heteroalbumose eine grössere als bei der Protalbumose, während bei der nach Kühne dargestellten und von Haslam untersuchten Heteroalbumose die Menge der Hexonbasen keinen derartigen Zuwachs zeigte. Beide Präparate, sowohl das von Pick wie auch das von Haslam, waren aus Witte-Pepton dargestellt.

Ich habe die Frage nach der Natur der Heteroalbumose und ihrem Verhältniss zur Protalbumose durch die Anwendung der oben erwähnten Methoden zur Bestimmung der Hexonbasen ihrer Lösung näher zu bringen versucht. Hierbei ergab sich eine Beobachtung in Bezug auf die Betheiligung des Lysins an der Bildung der Huminsubstanzen, welche mich veranlasste, die Bedingungen, unter denen der Stickstoff in den Huminsubstanzen gebunden wird, weiter zu verfolgen.

1) Diese Zeitschrift, Bd. XXVIII, S. 219.

2) Diese Zeitschrift, Bd. XXXII, S. 54. 
Da der Ursprung des käuflichen Witte-Peptons sich der Kontrolle entzieht, so stellte ich mir als Ausgangspunkt für meine Untersuchungen Syntonin aus Rindfleisch dar und gewann hieraus Protalbumose und Heteroalbumose.

I. Die Darstellung des Syntonins war folgende: Fein gehacktes mageres Rindfleisch wurde in weithalsige Flaschen gebracht, die mit Gaze zugebunden waren und so lange von Wasser durchströmt wurden, bis alles Blut herausgewaschen war. Nach dem Ablassen des Wassers liess ich es 24 Stunden unter gelegentlichem Umrühren in 0,1\% / iger Salzsäure (Liebig) ${ }^{1}$ ) stehen, dann durch leinene Beutel fliessen und filtrirte schliesslich durch Kattun. Hierdurch erhielt ich eine schwach gefärbte dicke Flüssigkeit. Nun wurde mit Soda neutralisirt, wobei sich das Syntonin in einer flockigen weissen Masse ausschied. Nachdem es sich $\mathrm{zu}$ Boden gesetzt hatte, wurde es durch zweimalige Decantation geklärt, dann filtrirt und mit Wasser gewaschen, hierauf abermals in 0,1\% iger Salzsäure gelöst und mit Soda niedergeschlagen, wiederum decantirt, filtrirt und mit Wasser gewaschen. So wurden gegen 1000 g Syntonin hergestellt, um theils zur Analyse, theils zu Verdauungsversuchen verwendet $\mathrm{zu}$ werden. Ich hielt es für sicherer, eine grosse Quantität herzustellen und durcheinander zu mischen, um für möglichst viele Untersuchungen dasselbe Präparat $\mathrm{zu}$ benutzen. Es wurde nun wieder in 0,1\% iger Salzsäure aufgelöst und nach Zusatz von Chloroform in Flaschen bei Seite gestellt. Ein Quantum von ungefähr $40 \mathrm{~g}$ wurde herausgenommen und sorgfältig mit $\mathrm{Na}_{2} \mathrm{CO}_{3}$ neutralisirt, filtrirt, erst mit Wasser, dann mit Alkohol gewaschen, mit Aether extrahirt und bei $110^{\circ}$ getrocknet, um dann durch Säure zerlegt zu werden.

II. Aus diesem Syntonin gewann ich Heteroalbumose nach folgendem Verfahren: Etwa $200 \mathrm{~g}$ Syntonin wurden auf einen Salzsäuregehalt von $0,2 \%$ gebracht, $500 \mathrm{ccm}$. eines frischen Extractes von Schweinemagenschleimhaut hinzugefügt und unter Zusatz von reichlich Chloroform in einem Brutschrank auf 38.0 erwärmt. Das Pepsinextract war folgendermassen hergestellt

1) Annalen der Chem. u. Pharm., Bd. 73, S. 125. 
worden: Die gereinigte Schleimhaut von Schweinemagen wurde fein zerhackt, mit $1 \frac{1 / 2}{2}$ Liter $0,2 \%$ iger Salzsäure behandelt und einige Stunden stehen gelassen, dann zu einer völlig klaren Lösung filtrirt und mit $3-4 \mathrm{ccm}$. Chloroform versetzt, um die Fäulniss $\mathrm{zu}$ verhindern. Es ging eine sehr intensive Pepsinverdauung vor sich, die ich eine Nacht hindurch wirken liess. Dann wurde sorgfältig mit $\mathrm{Na}_{2} \mathrm{CO}_{3}$ neutralisirt und filtrirt. Das klare Filtrat wurde 15 Stunden lang dialysirt, aber es entstand kein Niederschlag von Heteroalbumose. Erst als der ganze Inhalt der Dialysatoren vereinigt und bei $57-62^{\circ}$ eingedampft wurde, begann ein flockiger Niederschlag sich $\mathrm{zu}$ bilden. W'ährend die Lösung auf dieser Temperatur erhalten wurde, schied sich eine beträchtliche Menge von Eiweisssubstanz aus, bei höherer Temperatur trat hingegen keine weitere Abscheidung ein. Der durch Filtration von der Flüssigkeit getrennte Niederschlag wurde darauf zunächst mit Wasser - worin er völlig unlöslich war - , dann mit Alkohol und Aether gewaschen und über Schwefelsäure getrocknet. Die Ausbeute betrug etwa 3 g. Die Substanz war unzweifelhaft Heteroalbumose, die nicht durch Dialyse abtrennbar war, und zwar entweder weil sie in $\mathrm{zu}$ geringer Concentration vorhanden war, oder vielleicht weil andere Stoffe ihre Löslichkeit vermehren. Um die Annahme zu widerlegen, dass die erhaltene Substanz etwa nur nach dem Neutralisiren zurückgebliebenes Syntonin sei, wurde folgender Versuch angestellt: Ungefähr 20 g Syntonin wurden verdaut, bis sich nach der Neutralisation ein schwacher Niederschlag von rückständigem Syntonin ergab. Die von dem Niederschlag abfiltrirte Flüssigkeit wurde auf $65^{\circ}$ erhitzt; es trat aber keine Gerinnung ein, selbst nicht bei $80^{\circ}$. Hierdurch wurde bewiesen, dass, wenn die Verdauung eine lange Zeit hindurch wirkte und so genügend Gelegenheit gab, die primären Produkte in secundäre umzuwandeln, ein der Heteroalbumose entsprechender Niederschlag nicht zu bemerken war, selbst wenn ein Rest von Syntonin zurückblieb. Dass die Heteroalbumose vor der Dialyse nicht vollständig aus der Lösung abgeschieden wird, muss aus der im Filtrat nachträglich beim Erhitzen eintretenden Abscheidung geschlossen werden. Die 
Substanz war in verdünnter. Essigsäure löslich, während Syntonin unter. gleichen Verhältnissen gefällt wird. Letzteres gerinnt erst bei mehr als $70^{\circ}$, unser Präparat dagegen that dies schon bei $58-64^{\circ}$. Auch seine weisse gummiartige Beschaffenheit wich von dem gallertigen Aussehen des Syntonins ab. Getrocknet nahm es eine dunkelbraune Farbe an und war äusserst hart und spröde, Syntonin dagegen ist heller gefärbt und leicht in Pulver zu verwandeln. Die Ausbeute war so gering, dass das Verfahren wiederholt wurde, bis etwa $10 \mathrm{~g}$ dieses Stoffes beisammen waren. Es ergab die Reactionen von Molisch, Adamkiewicz und Millon.

III. Zum Vergleich wurde ein anderes Präparat dieser Heteroalbumose nach einer Pick's ${ }^{1}$ ) Verfahren ähnlichen Darstellungsweise gewonnen: Da mein gesammtes Syntonin zur Darstellung der geronnenen Heteroalbumose und der später zu beschreibenden Protalbumose verbraucht war, so wurde ein neues Quantum von $1000 \mathrm{~g}$ nach der oben auseinandergesetzten Methode hergestellt. Diese Menge wurde in 7 Liter 0,2\% iger Salzsäure gelöst, mit 500 ccm. kräftiger Pepsinlösung und $10 \mathrm{ccm}$. Chloroform versetzt und dann bei einer Temperatur von $38^{\circ}$ der Verdauung überlassen. Nun wurde sorgfältig mit $\mathrm{Na}_{2} \mathrm{CO}_{3}$ neutralisirt, filtrirt und das Filtrat bei $35^{\circ}$ im Vacuum zu einer etwa 40\% igen Lösung concentrirt, hierauf durch die doppelte Volumenmenge $96 \%$ igen Alkohols ein Niederschlag ausgefällt und eine Nacht über stehen gelassen. Darauf filtrirte ich und suspendirte die Substanz in $125 \mathrm{ccm}$. Wasser. Sie war in Wasser fast unlöslich, unterschied sich also hierin von Pick's ${ }^{2}$ ) aus Witte-Pepton gewonnener Heteroalbumose. Da sie nichts anderes als die der Heteroalbumose entsprechende Substanz sein konnte, so wurde sie jetzt nach Pick's Vorschrift mit dem gleichen Volumen gesättigter Ammoniumsulfatlösung behandelt, umgerührt, filtrirt und völlig trocknen gelassen, wieder vom Filter genommen, in $125 \mathrm{ccm}$. Wasser suspendirt, mit dem gleichen Volumen gesättigter Lösung von $\left(\mathrm{NH}_{4}\right)_{2} \mathrm{SO}_{4}$ behandelt, abermals filtrirt, in Wasser gebracht, mit dem

1) Diese Zeitschrift, Bd. XXVIII, S. 219.

2) Ebenda. 
halben Volumen 95\% \% igen Alkohols versetzt, filtrirt, noch einmal mit Alkohol behandelt und wiederum filtrirt. Aber das Auswaschen des Ammoniumsulfats mit 32\% igem Alkohol erwies sich als zu langwierig und unsicher. Daher wurde der Niederschlag in Wasser suspendirt, dialysirt, bis er frei von $\left(\mathrm{NH}_{4}\right)_{2} \mathrm{SO}_{4}$ war, und abermals auf ein Filter gebracht. Hiernach löste ich ihn in einer 5\% igen Kochsalzlösung auf und erhielt dadurch eine milchige . Flüssigkeit, die ich dann dialysirte. Dabei schied sich eine beträchtliche Menge aus, aber die Menge des Niederschlages nahm beim Erhitzen auf $60^{\circ}$ noch zu. Die Lösung, welche den Niederschlag enthielt, wurde nun bei $60^{\circ}$ eingedampft und nach dem Erkalten mit dem halben Volumen 96\% igen Alkohols behandelt, dann filtrirt, mit Aether gewaschen und über Schwefelsäure getrocknet. Die auf diese Weise hergestellte Substanz war vor dem Trocknen sehr wenig in Wasser löslich, nach dem Trocknen in 5\% iger Kochsalzlösung unlöslich. Aeusserlich ähnelte sie der durch das obige Verfahren (II) erhaltenen Heteroalbumose. Sie gab die Reactionen von Molisch, Adamkiewicz und Millon. Die Ausbeute betrug $11 \mathrm{~g}$.

IV. Die Darstellung der Protalbumose (Protosyntonose) wurde nach Folin's ${ }^{1}$ ) Methode ausgeführt. Das Filtrat von der durch Hitze coagulirten Heteroalbumose (siehe II) wurde concentrirt und dann vorsichtig gesättigte Kupferacetatlösung zugesetzt, bis sich kein weiterer Niederschlag mehr bildete. Nachdem derselbe sich abgesetzt hatte, wurde die überstehende Flüssigkeit abgegossen, der Rückstand in verdünnter Essigsäure gelöst (die Lösung ging äusserst schwierig von statten) und dann mit Natronlauge neutralisirt. Sobald der Niederschlag von Protalbumose $\mathrm{zu}$ erscheinen begann, wurde noch etwas Kupferacetat hinzugefügt. Hatte der Niederschlag sich dann abgesetzt, so wurde wieder decantirt und abermals in verdünnter Essigsäure gelöst, und dieser Process wurde noch zweimal wiederholt. Nachdem das Kupfer durch Schwefelwasserstoff ausgefällt war, wurde die Flüssigkeit eingedampft und in 96\% igen Alkohol gegossen. Dies ergab einen in Wasser leicht

1) Diese Zeitschrift, Bd. XXV, S. 152. 
löslichen Körper, der durch Natriumchlorid fällbar war und auch durch wenige Tropfen Salpetersäure ausgefällt wurde.

In nachstehender Tabelle sind die Procente von Stickstoff (nach der Methode von Kjeldahl), auf aschefreie Substanz bezogen, zusammengestellt mit dem Procentgehalt an Asche in den obigen Substanzen.

\begin{tabular}{r|r|r|r|r}
\hline & Syntonin & $\begin{array}{r}\text { Hetero- } \\
\text { albumose } \\
\text { nach Ver- } \\
\text { fahren II. }\end{array}$ & $\begin{array}{c}\text { Hetero- } \\
\text { albumose } \\
\text { nach Ver- } \\
\text { fahren II. }\end{array}$ & $\begin{array}{c}\text { Proto- } \\
\text { albumose }\end{array}$ \\
\hline Stickstoff auf aschefreie Sub- & & & & \\
stanz bezogen . . . . . & 15,89 & 16,09 & 16,12 & 15,88 \\
Asche . . . . . . . . . & 0,14 & 2,09 & 9,61 & 1,47 \\
\end{tabular}

Der hohe Aschegehalt der durch Alkoholfällung dargestellten Heteroalbumose beruhte auf einer unvollkommenen Entfernung des Kochsalzes, wie die genauere Untersuchung der Aschebestandtheile ergab. Diese zufällige Verunreinigung führte zu auffälligen Ergebnissen, welche mich veranlassten, den Einfluss des Kochsalzes auf den Gang der Spaltung bei einigen Eiweisskörpern zu verfolgen.

V. Spaltung durch Säuren. - Die Körper wurden alle auf folgende Weise zerspalten: $1 \mathrm{~g}$ Eiweisssubstanz wurde mit $3 \mathrm{~g}$ concentrirter Schwefelsäure und $6 \mathrm{~g}$ Wasser 14 Stunden lang im Sandbade unter einem Rückflusskühler gekocht. Die Methode für die Bestimmung der Hexonbasen und der Huminsubstanz war dieselbe wie die von Kossel und Kutscher ${ }^{1}$ ) bei ihren Studien über die Eiweisskörper benutzte, mit Ausnahme der Ammoniakbestimmung, für welche ich ein vereinfachtes Verfahren anwandte. Sie hatten nämlich dazu Magnesiumoxyd verwendet, aber diese Substanz musste nachher wieder entfernt werden, was beträchtliche Zeit und Mühe kostete. Im Baryumcarbonat nun fand ich eine Substanz, die nicht nur mit Leichtigkeit zur Bestimmung der übrigen Produkte wieder entfernt werden kann, sondern auch das gesammte Ammoniak liefert. Magnesiumoxyd gibt oft grössere Werthe als Baryum-

1) Diese Zeitschrift, Bd. XXXI, S. 16ă.

Hoppe-Seyler's Zeitschrift f. physiol. Chemie. XXXIII. 
carbonat, was seinen Grund wohl darin hat, dass es Ammoniak noch von anderen Körpern als Ammoniumsalzen losreisst. Zwar mag dies bei Baryumcarbonat ebenfalls in geringem Umfange der Fall sein, aber die niedrigeren Werthe, die es liefert, zeigen, dass es weniger kräftig wirkt, während es dabei doch alles Ammoniak ergibt.

Die in der folgenden Tabelle zusammengestellten Werthe ergeben eine Vorstellung von der Wirkungsweise des Baryumcarbonats im Verhältniss zur Magnesia. Der Ammonstickstoff ist in Procenten des Gesammtstickstoffs angegeben.

\begin{tabular}{l|c|c}
\hline & $\begin{array}{c}\text { Destillation } \\
\text { mit Magnesia }\end{array}$ & $\begin{array}{c}\text { Destillation } \\
\text { mit } \mathrm{BaCO}_{3}\end{array}$ \\
\hline Glutin . . . . . . . & 1,28 & 1,27 \\
Casein . . . . . . . & 10,22 & 8,87 \\
Syntonin . . . . . . & 7,13 & 4,32 \\
Ammoniumsulfat. . . . & 100 & 100
\end{tabular}

Diese Zahlen wurden nach der Zersetzung der Eiweisskörper mit concentrirter Salzsäure ( $1 \mathrm{~g}$ Substanz auf $5 \mathrm{ccm}$. $\mathrm{HCl}$ und 8 stündigem Kochen im Sandbade am Rückflusskühler erhalten. In den Fällen dagegen, wo Schwefelsäure zur Spaltung benutzt worden war, wurde bei der Ausführung der Bestimmung die Lösung der Endprodukte mit Barytwasser nahezu neutralisirt, filtrirt und der Niederschlag von $\mathrm{BaSO}_{4}$ und der Huminsubstanz dreimal mit Wasser ausgekocht und filtrirt. Die leicht angesäuerten Waschwässer wurden mit dem ersten Filtrat vermischt, dann wurden die Bestimmungen nach Kjeldahl ausgeführt. Zur Ammoniakbestimmung entnahm ich Quanta von je $100 \mathrm{ccm}$., versetzte dieselben mit Baryumcarbonat und destillirte daraus das Ammoniak ab, wobei ich Oxalsäure zum Absorbiren und Rosolsäure als Indicator benutzte. Es war nothwendig, das Destillat vor dem Titriren zu kochen, um Kohlensäure und andere flüchtige Körper, welche eine scharfe Endreaction verhindern, zu entfernen. Der nicht zur Ammonbestimmung verwendete Rest der Lösung wurde in einer Porzellanschale mit Baryumcarbonat zum Sieden erhitzt, 
um alles Ammoniak zu entfernen. Alle die verschiedenen von Ammoniak befreiten Flüssigkeitsmengen wurden nach der Destillation in die Porzellanschale zurückgebracht, mit Schwefelsäure angesäuert, filtrirt und der Niederschlag gut gewaschen. Die weitere Durchführung der Methode stimmte mit der von Kossel und Kutscher benutzten überein.

Die Resultate der Säurespaltung, wie sie mit der obigen Abänderung bei den vier Eiweisskörpern vorgenommen wurde, sind in nachstehender Tabelle angegeben.

\begin{tabular}{|c|c|c|c|c|c|c|c|c|c|c|}
\hline & \multicolumn{5}{|c|}{$\begin{array}{l}\text { Procente des Gesammt- } \\
\text { stickstoffs }\end{array}$} & \multicolumn{4}{|c|}{ Gewichtsprocente } & \multirow{2}{*}{$\begin{array}{c}\text { Zer- } \\
\text { setztes } \\
\text { Eiweiss } \\
\text { Gramm }\end{array}$} \\
\hline & $\begin{array}{c}\text { Histi- } \\
\text { din }\end{array}$ & Arginin & Lysin & $\left|\begin{array}{c}\text { Ammo- } \\
\text { niak }\end{array}\right|$ & $\left|\begin{array}{c}\text { Humin- } \\
\text { sabstanz }\end{array}\right|$ & $\left|\begin{array}{c}\text { Hists- } \\
\text { din }\end{array}\right|$ & \begin{tabular}{|c|}
$\begin{array}{c}\text { Irgi- } \\
\text { nin }\end{array}$ \\
\end{tabular} & Lysin & \begin{tabular}{|c|} 
Immo- \\
nlak
\end{tabular} & \\
\hline Syntonin & 4,53 & 10,29 & 3,98 & 4,32 & 8,34 & 2,66 & 5,06 & 3,26 & 0,83 & 46,4 \\
\hline $\begin{array}{l}\text { Heteroalbumose (durch } \\
\text { Coagulation) } . \cdot \cdot \text {. }\end{array}$ & 1,92 & 17,46 & 3,76 & 2,92 & 11,65 & 1,12 & 8,55 & 3,08 & 0,56 & 7,1 \\
\hline $\begin{array}{l}\text { Heteroalbumose (durch } \\
\text { Alkoholfällung) } \cdot \text {. }\end{array}$ & 0,64 & 17,36 & 8,11 & $4,9 \tilde{5}$ & 6,80 & 0,37 & 8,52 & 7,03 & 0,97 & 8,2 \\
\hline Protalbumose $\cdot$. . & 5,76 & 9,30 & 3,76 & 4,01 & 9,17 & 3,35 & 4,55 & 3,08 & 0,76 & 12,2 \\
\hline
\end{tabular}

Bei Betrachtung dieser Zahlen sieht man, dass die Protalbumose sich wenig von der Mutter-Eiweisssubstanz (Syntonin) unterschied, während beide Heteroalbumosen sowohl von dem Syntonin wie auch von der Protalbumose erheblich verschieden waren - und dass ausserdem zwischen den beiden Heterosyntonosen selbst ein Unterschied bestand. Zählt man die in den Hexonbasen, im Ammoniak und in den Huminsubstanzen enthaltenen Stickstoffprocente zusammen, so ergeben sich folgende Zahlen:

Syntonin . . . . . . . . . . . $31,46 \%$
Heterosyntonose durch Coagulation . . . . $37,71 \%$
Heterosyntonose durch Alkoholfällung. . . . $37,86 \%$
Protosyntonose . . . . . . . . - $32,00 \%$.

Es ergab sich somit der auffallende Befund, dass die Summe der Stickstoffprocente in den beiden HeterosyntonosePräparaten die gleiche war. Bei dem einen dieser Präparate war weit weniger Huminstickstoff vorhanden, doch war das 
Deficit an Huminstickstoff durch ein Plus an Lysin und Ammoniak wieder ausgeglichen:

Wie oben erwähnt, enthielt die durch Alkohol gefällte. Heteroalbumose viel Kochsalz; es enstand nun die Frage, ob die Gegenwart des Kochsalzes etwa einen Einfluss auf das quantitative Ergebniss der Spaltung besitze. Zur Entscheidung dieser Frage diente die folgende Untersuchung des Caseins.

VI. Spaltung des Caseins. Ein von Merck bezogenes Präparat von Casein wurde in oben beschriebener Weise der Zersetzung mit Schwefelsäure unterworfen. Der Versuch wurde sodann in gleicher Weise wiederholt mit der einzigen Abänderung, dass $3 \mathrm{~g}$ Kochsalz hinzugefügt wurden.

Bei der Berechnung der Stickstoffprocente ist der von. Chittenden gefundene Procentgehalt des Caseins von $15,91 \% \mathrm{~N}$ zu Grunde gelegt worden.

Spaltung des Caseins. (Versuch I.)

\begin{tabular}{|c|c|c|c|c|c|c|c|c|c|c|}
\hline & \multicolumn{5}{|c|}{$\begin{array}{l}\text { Procente des Gesammt- } \\
\text { stickstoffs }\end{array}$} & \multicolumn{4}{|c|}{ Gewichtsprocente } & \multirow{2}{*}{$\begin{array}{c}\text { Zer- } \\
\text { setztes } \\
\text { Eiweiss } \\
\text { Gramm }\end{array}$} \\
\hline & $\begin{array}{c}\text { Histi- } \\
\text { din }\end{array}$ & $\begin{array}{c}\text { Argi- } \\
\text { nin }\end{array}$ & Lysin & $\begin{array}{c}1 \text { mmo- } \\
\text { niak }\end{array}$ & $\left|\begin{array}{c}\text { Humin- } \\
\text { substanz }\end{array}\right|$ & $\begin{array}{c}\text { Histi- } \\
\text { din }\end{array}$ & $\mid \begin{array}{c}\text { Argi- } \\
\text { nin }\end{array}$ & Lysin & $\mid \begin{array}{c}\text { Immo- } \\
\text { niak }\end{array}$ & \\
\hline Ohne Kochsalz & 3,66 & 9,51 & 2,31 & 7,34 & 9,09 & 2,53 & 4,70 & 1,92 & 1,43 & 9,7 \\
\hline Mit Kochsalz & 3,75 & 9,80 & 6,98 & 9,48 & 1,77 & 2,59 & 4,84 & 5,80 & 1,84 & 9,2 \\
\hline
\end{tabular}

Hier bemerkt man wieder, dass durch die Gegenwart von Chlornatrium der Stickstoff der Huminsubstanz verringert und statt dessen die Menge des Ammoniaks und des Lysins vermehrt war; ferner, dass die Summen der bestimmten Bestandtheile in Procenten des Gesammtstickstoffs 31,91 resp. 31,88 , also annähernd gleich sind. Hier besteht also dieselbe Beziehung, wie bei den beiden Heteroalbumosen. Diese Beobachtungen können den Gedanken nahelegen, dass derjenige Stickstoff, welcher bei der Spaltung in Gegenwart von Kochsalz an Lysin und Ammoniakstickstoff mehr gefunden wird, bei Abwesenheit von Kochsalz in Huminstickstoff übergeht. 
Bei einem zweiten Versuch, in welchem nur Lysin und Ammoniak bestimmt wurde, zeigte sich der Einfluss des Kochsalzes in gleichem Sinne, doch waren die Unterschiede geringer.

Spaltung des Caseins. (Versuch II.)

\begin{tabular}{c|c|c|c|c|c}
\hline & \multicolumn{2}{|c|}{$\begin{array}{c}\text { Procente des } \\
\text { Gesammtstickstoffs }\end{array}$} & \multicolumn{2}{c|}{ Gewichtsprocente } & $\begin{array}{c}\text { Zersetztes } \\
\text { Eiweiss. }\end{array}$ \\
\cline { 2 - 6 } & Lysin & Ammoniak & Lysin & Ammoniak & \\
\hline Ohne Kochsalz ... & 5,3 & 6,7 & 4,4 & 1,29 & 9,51 \\
Mit Kochsalz .... & 6,9 & 8,1 & 5,7 & 1,57 & $9,5 \dot{0}$
\end{tabular}

In einer anderen durch Schwefelsäure in Gegenwart von Kochsalz zerspaltenen Probe von Casein, wobei nur die Huminsubstanz und der Ammoniakgehalt bestimmt wurden, war der Stickstoff in der Huminsubstanz vollständig verschwunden, während er in dem Ammoniak auf 10,12\% gestiegen war.

VII. Spaltung des Leims. Hiernach wurde Leim untersucht, um einerseits die Hexonbasen; Ammoniak und Huminsub'stanz zu bestimmen, die bei einer Spaltung durch Säure, wie sie theilweise von Kossel und Kuts $\mathrm{Kher}^{1}$ ) untersucht wurde, resultiren, und um andererseits festzustellen, ob hier wieder durch die Gegenwart von Chlornatrium der Stickstoff ganz oder theilweise von der Huminsubstanz losgerissen und Ammoniak und Lysin gebildet würde. Diè Darstellung geschah ungefähr nach der Methode von Mörner. ${ }^{2}$ ) Etwa $300 \mathrm{~g}$ gute käufliche Gelatine wurden fein zerschnitten und 5 Tage lang unter häufigem Umrühren und Wasserwechsel eingeweicht, wobei das Wasser mit Chloroform gesättigt erhalten wurde. Hierauf wurde das Wasser abgelassen, der Rest dann mit 1\% iger Lösung von $\mathrm{KOH}$ behandelt und zwei Tage unter häufigem Umrühren stehen gelassen. Darauf wurde die Masse in leinene Beutel gebracht und erst mit destillirtem Wasser, dann mit verdünnter Essigsäure und danach wieder mit Wasser tüchtig gewaschen, hierauf

1) Diese Zeitschrift, Bd. XXXI, S. 165.

2) Diese Zeitschrift, Bd. XXXVIII, S. 472. 
in Standgläsern unter Alkohol gehärtet, nach dem Ablassen desselben in Porzellanschalen im Wasserbade geschmolzen, durch Kattun filtrirt und abermals mit Alkohol ausgefällt. Eine kleine Probe wurde mit Alkohol und Aether gewaschen und bei $110^{\circ}$ getrocknet. Die Bestimmung nach Kjeldahl ergab einen Stickstoffgehalt von 17,54\% (nicht auf aschefreie Substanz bezogen). Die Millon'sche Reaction war deutlich, aber schwach, wodurch die Beobachtung von Mörne ${ }^{1}$ ) über diesen Punkt bestätigt wird. Der Rest wurde über Schwefelsäure getrocknet, dann wurden etwa $50 \mathrm{~g}$ davon 14 Stunden lang im Sandbade am Rückflusskühler mit $150 \mathrm{~g}$ concentrirter Schwefelsäure und $300 \mathrm{~g}$ Wasser gekocht.

Ein anderes Quantum von gutem käuflichen Leim wurde zur Spaltung in Gegenwart von Kochsalz benutzt. Etwa $12 \mathrm{~g}$ wurden mit $3 \mathrm{~g} \mathrm{NaCl}, 36 \mathrm{~g} \mathrm{H}_{2} \mathrm{SO}_{4}$ und $72 \mathrm{~g} \mathrm{H}_{2} \mathrm{O} 14$ Stunden lang gekocht. Bei dieser Spaltung wurden das Arginin und das Histidin nicht bestimmt. Die Ergebnisse sind in nachstehender Tabelle zusammengestellt.

Spaltung des Leims.

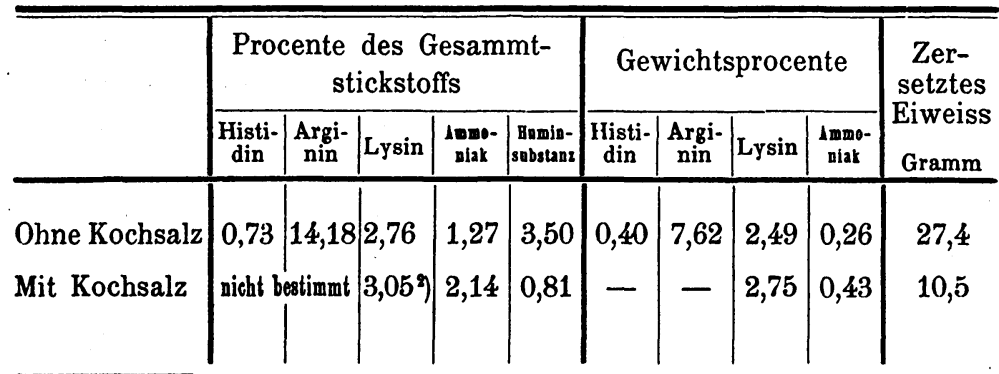

1) Diese Zeitschrift, Bd. XXXVIII, S. 472.

2) Um festzustellen, ob das Lysin, als Pikrat gewogen, rein war, wurde es vollständig in Hydrochlorid umgewandelt und in der ganzen eingedampften Masse Chlor und Stickstoff bestimmt. Das Resultat war folgendes :

Berechnet: für $\mathrm{C}_{6} \mathrm{H}_{14} \mathrm{~N}_{2} \mathrm{O}_{2}, 2 \mathrm{HCl}$.

N $\quad 12,79 \%$

Cl $32,39 \%$
Gefunden:

12,54

32,20

Das bei Zusatz von Kochsalz erhaltene Lysin erwies sich somit als völlig rein. 
Die Zahlen zeigen, dass die Gegenwart von $\mathrm{NaCl}$ während der Spaltung auch hier eine Abnahme des Stickstoffs in der Huminsubstanz und eine Zunahme desselben in Ammoniak und Lysin zur Folge hatte.

VIII. Der Erhöhung des Siedepunktes ist dieser Einfluss des Kochsalzes nicht zuzuschreiben; denn der Siedepunkt von $60 \mathrm{~g} \mathrm{H}_{2} \mathrm{O}+30 \mathrm{~g} \mathrm{H}_{2} \mathrm{SO}_{4}$ liegt $z$ wischen 109 und $110^{\circ}$ und wird durch Zusatz von $3 \mathrm{~g} \mathrm{NaCl}$ nicht in erheblicher Weise erhöht, wie mit einem gewöhnlichen Thermometer festgestellt wurde. Natriumsulfat, das bei einer Spaltung von $12 \mathrm{~g}$ Casein an die Stelle des Natriumchlorids gesetzt wurde, hatte dieselbe Wirkung, indem es den Stickstoff der Huminsubstanz auf 1,75\% des Gesammtstickstoffs reducirte; wenn es dagegen durch $4 \mathrm{ccm}$. $1,19 \%$ iger Salzsäure ersetzt wurde, so trat nur eine Reduction auf $4,34 \%$ ein.

Udransky ${ }^{1}$ ) war es gelungen, durch Zersetzung .von Zucker, bei Gegenwart von Harnstoff, Huminsubstanz herzustellen, welche Stickstoff enthielt. Es schien von Interesse, diesen Versuch mit Lysin an Stelle des Harnstoffs zu wiederholen. Ich kochte deshalb ein Gemisch von $2 \mathrm{~g}$ Lysin, $10 \mathrm{~g}$ Rohrzucker, $30 \mathrm{~g}$ Schwefelsäure und $60 \mathrm{~g}$ Wasser 8 Stunden hindurch. Es schied sich eine schwarze Masse aus, die von der Flüssigkeit abfiltrirt, gut mit Wasser gewaschen und über Schwefelsäure getrocknet wurde. Eine Untersuchung diéses Rückstandes auf Stickstoff zeigte, dass er von diesem Elemente frei war.

IX. Spaltung von Zein. Kossel und Kutscher²) haben gezeigt, dass die in Alkohol löslichen Pflanzeneiweisskörper bei der Säurespaltung kein Lysin hergaben. Es war die Frage, ob diese Stoffe Lysin liefern würden, wenn sie in Gegenwart von Kochsalz zersetzt werden. Um dies festzustellen, wurde zunächst Zein aus ganzen Getreidekörnern nach der Methode von Ritthausen $^{3}$ ) hergestellt, dann wurde es mit $30 \mathrm{~g}$ concentrirter

1) Diese Zeitschrift, Bd. XII, S. 33.

2) Diese Zeitschrift, Bd. XXXI, S. 165.

3) Die «Eiweisskörper» der Getreidearten etc. Bonn 1872. 
Schwefelsäure, $3 \mathrm{~g}$ Kochsalz und $60^{\circ} \mathrm{g}$ Wasser im Sandbad am Rückflusskühler gekocht. Folgendes sind die in Procenten des Gesammtstickstoffs gegebenen Resultate, verglichen mit denen von Kossel und Kutscher:

\begin{tabular}{l|c|c}
\hline & $\begin{array}{c}\text { Zein } \\
\text { ohne Kochsalz zersetzt } \\
\text { Kossel u. Kutscher }\end{array}$ & $\begin{array}{c}\text { Zein 1) } \\
\text { bei Gegenwart von } \\
\text { Kochsalz zersetzt }\end{array}$ \\
\hline Lysin . . . . . & Kein Stickstoff & Kein Stickstoff. \\
Ammoniak . . . . & 13,53 & $\begin{array}{c}17,21 \\
\text { Huminsubstanz . . }\end{array}$ \\
\hline 11,83 & Kein Stickstoff.
\end{tabular}

Man sieht hier, dass bei dem Kochsalzversuch ein Theil des der Huminsubstanz angehörigen Stickstoffs zur Bildung von Ammoniak verwandt war, dass aber der übrige, ebenfalls verschwundene Huminstickstoff kein Lysin gebildet hatte. Das Lysin ist also nicht etwa die einzige Quelle des Huminstickstoffs. Jedenfalls ist es von grossem Interesse, dass hier aus einem Eiweisskörper, dem Zein, eine stickstofffreie Huminsubstanz wie aus einem Kohlehydrat hervorgegangen war.

$\mathrm{X}$. Da ich genöthigt war, meine Arbeiten abzubrechen, so ist es mir nicht möglich gewesen, meine Untersuchungen über diesen Einfluss des Kochsalzes auf die Säurespaltung bei anderen Eiweisskörpern zum Abschluss zu bringen oder weitere Aufklärungen über diese Einwirkung zu erlangen. Ich habe nur noch eine Frage in den Kreis meiner Arbeiten hineinziehen können: ob vielleicht in den stickstoffhaltigen Huminsubstanzen, welche aus Eiweiss hervorgehen, die Gruppe des Lysins noch in der Weise enthalten ist, dass aus ihnen Lysin gewonnen werden kann. Daher wurde Huminsubstanz selbst durch Spaltung von Casein mit concentrirter Salzsäure hergestellt, um zu ermitteln, ob, nachdem sie einmal gebildet war, ihr Stickstoff durch Kochen mit Schwefelsäure in Gegenwart von Kochsalz wieder losgelöst werden könnte, um dann Ammoniak und Lysin zu bilden. $125 \mathrm{~g}$ Merck'sches Casein

1) $11,8 \mathrm{~g}$ zersetzt. 
wurden mit $500 \mathrm{ccm}$. concentrirter Salzsäure acht Stunden lang im Sandbade am Rückflusskühler gekockt. Die dunkelgefärbte Lösung, die einen geringen schwarzen, unlöslichen Rückstand enthielt, wurde hierauf vorsichtig mit $\mathrm{Ba}(\mathrm{OH})_{2}$ versetzt, bis es auf Lakmus nur schwach sauer reagirte, wobei ein beträchtlicher Niederschlag sich zu bilden begann, der die Lösung beinahe ganz entfärbte. Dies war die zu untersuchende Huminsubstanz. Die Flüssigkeit wurde nun in Standgläser gegossen und, nachdem der Niederschlag sich zu Boden gesetzt hatte, derselbe so lange durch abwechselnde Decantation ausgewaschen, bis die Waschflüssigkeit mit Phosphorwolframsäure keine Trübung mehr gab, wodurch die vollständige Entfernung aller Hexonbasen verbürgt war. Dann wurde filtrirt, mit Wasser gewaschen und über Schwefelsäure getrocknet. Ein Theil dieser schwarzen, amorphen Masse wurde 8 Stunden lang mit $24 \mathrm{~g}$ concentrirter Schwefelsäure, $48 \mathrm{~g}$ Wasser und $3 \mathrm{~g}$ Kochsalz im Sandbade am Rückflusskühler gekocht. Der gesammte Stickstoffgehalt der Lösung betrug 0,161 g. Nun wurde die Lösung mit $\mathrm{Ba}(\mathrm{OH})_{2}$ behandelt, bis sie nur noch schwach sauer reagirte, dann filtrirt und der Niederschlag von Baryumsulfat gut gewaschen. Das Filtrat enthielt $0,28 \mathrm{~g}$ Stickstoff, das heisst, 17,3\% des Gesammtstickstoffs waren losgetrennt worden in einer Gestalt, die in schwach saueren Lösungen löslich war. Eine Ammoniakbestimmung, die durch Destillation mit $\mathrm{BaCO}_{3}$ an diesem löslichen Stickstoff vorgenommen wurde, ergab $0,0043 \mathrm{~g} \mathrm{~N}$ oder 2,64\% des Gesammtstickstoffs. Nun wurde die Lösung mit Schwefelsäure angesäuert, filtrirt, gewaschen und concentrirt. Die schwach bräunlich gefärbte Lösung wurde nun mit Schwefelsäure auf $5 \%$ angesäuert und Phosphorwolframsäure hinzugefügt; es trat ein leichter flockiger bräunlicher Niederschlag auf, der die Lösung vollständig entfärbte. Die Untersuchung dieses Niederschlages auf Lysin ergab negative Resultate. Nachdem der ungelöste Theil der Huminsubstanz bis zur Gewichtsconstanz über Schwefelsäure getrocknet war, wurde sie selbst auf Stickstoff untersucht, wobei sich $1,34 \%$ ergaben. 


\section{Zusammenfassung einiger Ergebnisse.}

1. Die beiden bei der Pepsinverdauung des Syntonins gebildeten primären Produkte unterscheiden sich in ihrer Structur durch den Gehalt an Hexonbasen von einander und yon der Muttersubstanz, dem Syntonin. Die Heteroalbumose enthält weniger, die Protalbumose mehr Histidin. Hingegen ist die Heteroalbumose reicher an Arginin als die Protalbumose.

2. Die beiden nach verschiedenen Methoden (Coagulation, Alkoholfällung) dargestellten Präparate von Heteroalbumose sind wahrscheinlich identisch. Der bezüglich des Lysins, des Ammoniaks und des Huminstickstoffs gefundene Unterschied ist wahrscheinlich dadurch herbeigeführt worden, dass das eine Präparat mehr Kochsalz enthielt als das andere.

3. Wird die Spaltung von Casein oder Leim durch siedende Säuren bei Gegenwart einer gewissen Menge gelöster anorganischer Salze (Kochsalz, Natriumsulfat) vorgenommen, so kann die Spaltung anders verlaufen als bei Abwesenheit dieser Salze. Bei Gegenwart von Kochsalz war bei diesen Eiweisskörpern die Menge des gebildeten Ammoniaks und des Lysins eine grössere, die des Huminstickstoffs eine geringere.

4. Aus einzelnen Eiweisskörpern gelingt es, durch Säurespaltung bei Gegenwart von Kochsalz stickstofffreie Huminsubstanzen $\mathrm{zu}$ erhalten.

5. Das Zein liefert, auch bei Gegenwart von Kochsalz gespalten, kein Lysin.

6. Die einmal aus Casein gebildete Huminsubstanz liefert bei nachträglicher Spaltung mit Schwefelsäure und Kochsalz kein Lysin mehr.

Ich möchte die Gelegenheit ergreifen, an dieser Stelle Herrn Prof. Dr. Kossel meinen aufrichtigen Dank auszusprechen für die Anregung zu dieser Arbeit und für die stetige Aufmerksamkeit und Hilfe, die er mir während der Ausführung der obigen Untersuchung hat zu Theil werden lassen. 